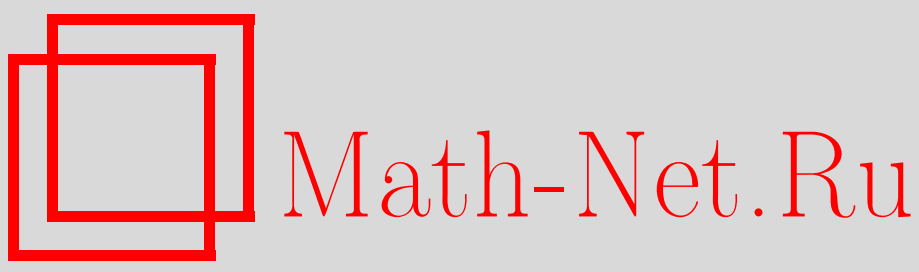

Д. А. Шакин, О некоторых обобщениях комбинаторной теоремы Маколея на случай факторколец, Матем. сб., 2001, том 192, номер 9, 143-160

DOI: https://doi.org/10.4213/sm598

Использование Общероссийского математического портала Math-Net.Ru подразумевает, что вы прочитали и согласны с пользовательским соглашением http://www . mathnet.ru/rus/agreement

Параметры загрузки:

IP: 35.173 .219 .12

26 апреля 2023 г., 12:38:37 


\title{
О некоторых обобщениях комбинаторной теоремы Маколея на случай факторколец
}

\begin{abstract}
В настоящей статье рассматривается задача описания функций Гильберта однородных идеалов в колње многочленов, содержащих мономиальный идеал $I$. Полученное описание функций Гильберта обобщает результат Маколея для кольца многочленов на случай факторколец по некоторьм мономиальньм идеалам. В частности, приведено необходимое и достаточное условие на идеал $I$ для выполнения теоремы Маколея в факторколце кольца многочленов от двух переменных, а также если $I$ является лексическим. Также доказьвается возможность обобщения теоремы Маколея для широкого класса идеалов, порожденных мономами от первых по порядку двух переменных. Кроме того, приводится эквивалентная формулировка теоремы Маколея и необходимые условия на идеал $I$, при которых обобщение возможно.

Библиографояи: 4 названия.
\end{abstract}

\section{§1. Введение}

Пусть $A=k\left[x_{1}, x_{2}, \ldots, x_{n}\right]$ - кольцо многочленов от $n$ переменных над полем $k$ со стандартной градуировкой, т.е. $\operatorname{deg} x_{i}=1 \forall i$. Пусть $J$ - однородньй идеал и $H(J, d)=\operatorname{dim}_{k} J_{d}-$ его функция Гильберта.

В 1927 году Маколеем [1] доказана теорема, из которой следует, что функции Гильберта однородных идеалов $J \subseteq A$ исчерпываются функциями Гильберта лекс-сегментных идеалов, т.е. мономиальных идеалов, состояших в каждой степени из старших по лексикографическому порядку мономов:

ТЕОРема 1.1 (Маколей). Для любого однородного идеала $J$ существует лекс-сегментный идеал $L$ такой, что $H(J, d)=H(L, d) \forall d \geqslant 1$.

Пусть $\mathfrak{A}$ - множество всех однородных идеалов в $A, \mathfrak{B}$ - некоторое его подмножество, $H(\mathfrak{B})$ - множество функций Гильберта идеалов $J \in \mathfrak{B}$. В настояшей статье рассматривается следуюшая задача: найти описание множества $H(\mathfrak{B})$, аналогичное полученному Маколеем, если в качестве $\mathfrak{B}$ рассмотреть множество всех идеалов из $\mathfrak{A}$, содержащих некоторый мономиальньй идеал $I$. Более конкретно: каким условиям должен удовлетворять идеал $I$ для того, чтобы было справедливо равенство $H(\mathfrak{B}(I))=H\left(\mathfrak{B}_{0}(I)\right)$ для некоторого $\mathfrak{B}_{0}(I) \subseteq \mathfrak{B}(I)$. В качестве $\mathfrak{B}_{0}(I) \subseteq \mathfrak{B}(I)$ естественно взять множество идеалов из $\mathfrak{B}(I)$, содержащих, кроме мономов из $I$, старшие мономы в каждой степени. В частности, теорема Маколея 1.1 утверждает, что при $I=0\left(\mathfrak{B}(I)=\mathfrak{A}, \mathfrak{B}_{0}(I)\right.$ - множество

Работа выполнена при частичной поддержке Российского фонда фундаментальных исследований (грант № 99-01-01144).

(C) Д. А. ШАКин 2001 
всех лекс-сегментных идеалов) подобное описание возможно. Авторы работы [2] обобшили результат Маколея на случай, когда $I=\left(x_{1}^{d_{1}}, x_{2}^{d_{2}}, \ldots, x_{n}^{d_{n}}\right) A$, причем $d_{1} \leqslant d_{2} \leqslant \cdots \leqslant d_{n} \leqslant \infty$.

Следует отметить, что случай произвольного однородного идеала $I$ нельзя свести к случаю, рассматриваемому в нашей статье:

ПримеР 1.2. Пусть $f\left(x_{1}, \ldots, x_{n}\right) \in A=k\left[x_{1}, \ldots, x_{n}\right]$ - однородный многочлен степени $d \geqslant 2$, неприводимый над полем $k, I=(f) A-$ однородный идеал. Тогда для любого мономиального идеала $K \subseteq A$ множества $H(\mathfrak{B}(I))$ и $H(\mathfrak{B}(K))$ различны.

Действительно, рассмотрим произвольный мономиальньй идеал $K \subseteq A$. Предположим, что $H(\mathfrak{B}(I))=H(\mathfrak{B}(K))$. Тогда, в частности, $H(I, n)=H(K, n) \forall n$, следовательно, $H(I, d-1)=H(K, d-1)=0, H(I, d)=H(K, d)=1$. Так как $K-$ мономиальный идеал, то $K_{d}$ порождено одним мономом $m$ степени $d$. Рассмотрим идеал $J=\left(m / x_{i}, K\right) A \in \mathfrak{B}(K)$ для некоторого $i$ такого, что $x_{i}$ делит $m$. Очевидно, что $H(J, d-1)=1, H(J, d)=n$. Так как $H(\mathfrak{B}(I))=H(\mathfrak{B}(K))$, то $\exists M \in \mathfrak{B}(I)$ такой, что $H(M, d-1)=1, H(M, d)=n$. Следовательно, $M_{d-1}$ порождено одним однородным многочленом $g$ степени $d-1$, но в силу неприводимости $f$ многочлены $f, g x_{1}, g x_{2}, \ldots, g x_{n}$ линейно независимы над $k$, т.е. $H(M, d)=n+1$ - противоречие.

С другой стороны, случай произвольного мономиального идеала $I$ нельзя свести к случаю лекс-сегментного идеала:

ПримеР 1.3. Пусть $A=k\left[x_{1}, x_{2}\right], I=\left(x_{1}^{2}, x_{2}^{3}\right) A$-мономиальный идеал. Тогда для любого лекс-сегментного идеала $L$ множества $H(\mathfrak{B}(I))$ и $H(\mathfrak{B}(L))$ различњы.

Действительно, рассмотрим произвольный лекс-сегментньй идеал $L \subseteq A$. Предположим, что $H(\mathfrak{B}(I))=H(\mathfrak{B}(L))$. Тогда, в частности, $H(I, n)=H(L, n)$ $\forall n$, следовательно, $L=\left(x_{1}^{2}, x_{1} x_{2}^{2}, x_{2}^{4}\right) A$. Положим $J=\left(x_{1} x_{2}, L\right) A \in \mathfrak{B}(L)$. Очевидно, что $H(J, 2)=2, H(J, 3)=3$. Однако $\forall M \in \mathfrak{B}(I)$ из $H(M, 2)=2$ следует, что $H(M, 3)=4$ - противоречие.

Заметим сразу, что изучение функций Гильберта однородных идеалов $J \in \mathfrak{B}(I)$ можно свести к изучению функций Гильберта мономиальных идеалов в силу следующей теоремы.

ТЕорема 1.4 (Маколей). Функиия Гильберта однородного идеала J совпадает с функиией Гильберта мономиального идеала стариих членов іп $J$.

(Остается заметить, что если $J \in \mathfrak{B}(I)$, то in $J \in \mathfrak{B}(I)$, так как $I$ мономиальный.) Таким образом, не ограничивая общности, можно рассматривать только мономиальные идеалы $J \in \mathfrak{B}(I)$.

Везде далее в статье: $A=k\left[x_{1}, x_{2}, \ldots, x_{n}\right]$ - кольцо многочленов от $n$ переменных, $I$ - некоторый мономиальный идеал в $A, R=A / I$ - факторкольцо, $A_{i}, R_{i}$, $I_{i}$ - множества мономов степени $i$ в $A, R, I$ соответственно. Введем на мономах в $A$ лексикографический порядок: $x_{1}^{a_{1}} \cdots x_{n}^{a_{n}}>x_{1}^{b_{1}} \cdots x_{n}^{b_{n}}$, если $a_{1}=b_{1}, a_{2}=b_{2}$, $\ldots, a_{l}=b_{l}, a_{l+1}>b_{l+1}$. Так как $I$ - мономиальньй идеал, то на мономах в $R$ индуцируется аналогичный порядок.

Теорему Маколея можно сформулировать в терминах факторкольца $R$ : требование $J \in \mathfrak{B}(I)$ означает, что рассматриваются образы однородных идеалов из $\mathfrak{A}$ в 
факторкольце $R=A / I$, а $\mathfrak{B}_{0}(I)$-множество мономиальных идеалов в $R$, содержащих старшие мономы в каждом $R_{i}$. Удобно переформулировать теорему Маколея в комбинаторных терминах. Пусть $M$ - мономиальное множество в $R_{i}$, обозначим через $D^{R} M$ множество, состояшее из старших мономов в $R_{i},\left|D^{R} M\right|=|M|$, а через $C^{R} M$ - множество, состоящее из меньших мономов в $R_{i},\left|C^{R} M\right|=|M|$ (верхний индекс ${ }^{R}$ указывает, в каком кольце действуют операторы $C, D$ ). Если $a=x_{1}^{a_{1}} \cdots x_{n}^{a_{n}} \in R_{i}$, то обозначим

$$
\begin{gathered}
\Gamma^{R}(a)=\left\{x_{1}^{a_{1}-1} x_{2}^{a_{2}} \cdots x_{n}^{a_{n}}, x_{1}^{a_{1}} x_{2}^{a_{2}-1} \cdots x_{n}^{a_{n}}, \ldots, x_{1}^{a_{1}} x_{2}^{a_{2}} \cdots x_{n}^{a_{n}-1}\right\} \cap R_{i-1}, \\
\Gamma^{R}(M)=\bigcup_{a \in M} \Gamma^{R}(a), \\
\Delta^{R}(a)=\left\{x_{1}^{a_{1}+1} x_{2}^{a_{2}} \cdots x_{n}^{a_{n}}, x_{1}^{a_{1}} x_{2}^{a_{2}+1} \cdots x_{n}^{a_{n}}, \ldots, x_{1}^{a_{1}} x_{2}^{a_{2}} \cdots x_{n}^{a_{n}+1}\right\} \cap R_{i+1}, \\
\Delta^{R}(M)=\bigcup_{a \in M} \Delta^{R}(a) .
\end{gathered}
$$

Будем называть множество $M \subseteq R_{i} D^{R}$-сжсатым, если $D^{R} M=M$, и $C^{R}$-сжаmылм, если $C^{R} M=M$.

Теорема Маколея 1.1 утверждает, что

$$
\left|\Delta^{A}\left(D^{A} M\right)\right| \leqslant\left|\Delta^{A}(M)\right| \forall i \geqslant 1, \quad \forall M \subseteq A_{i} .
$$

Будем говорить, что в факторкольце $R$ выполняется теорема Маколея, если

$$
\left|\Delta^{R}\left(D^{R} M\right)\right| \leqslant\left|\Delta^{R}(M)\right| \forall i \geqslant 1, \quad \forall M \subseteq R_{i}
$$

Легко видеть, что (1) эквивалентно обобщению теоремы Маколея, приведенному выше.

Основной целью статьи является нахождение условий на идеал $I$, при которых в кольце $R$ выполняется теорема Маколея (1).

В $\S 2$ статьи изложены, в основном, технические утверждения, в том числе равносильная формулировка теоремы Маколея (теорема 2.7) и два условия на идеал $I$, необходимых для выполнения в $R$ теоремы Маколея (следствие 2.5 и теорема 2.10).

В $\S 3$ приведено условие на идеал $I$, необходимое и достаточное для выполнения теоремы Маколея в кольце $R$, если $A=k\left[x_{1}, x_{2}\right]$ (теорема 3.5$)$. Кроме того, с помощью метода, изложенного в [2] (с небольшими изменениями), получено обобщение теоремы Маколея на факторкольца по некоторьм идеалам, порожденным мономами от переменных $x_{1}$ и $x_{2}$ (теорема 3.1 ).

В $\S 4$ изложены условия, необходимые и достаточные для выполнения теоремы Маколея, если $I$ является лексическим (см. определение в 4 ) или вполне лексическим (теоремы 4.12, 4.11).

Автор хотел бы выразить свою глубокую признательность Е. С. Голоду за его многочис ленные ценные замечания. 


\section{§2. Свойства сжатых множеств и отображений $\Gamma$ и $\Delta$}

ЛЕмма 2.1. Если $I$ - произвольный мономиальный идеал, $M \subseteq R_{i}-$

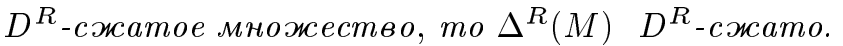

ДокАЗАТЕЛЬСтво. Пусть $\Delta^{R}(M)$ не $D^{R}$-сжато, т.е. существуют

$$
a=x_{1}^{a_{1}} \cdots x_{n}^{a_{n}} \in R_{i+1} \backslash \Delta^{R}(M), \quad b=x_{1}^{b_{1}} \cdots x_{n}^{b_{n}} \in \Delta^{R}(M)
$$

такие, что $a>b$. Так как $a>b$, то $\exists l \geqslant 1$ такое, что $a_{1}=b_{1}, \ldots, a_{l-1}=b_{l-1}$, $a_{l}>b_{l}$, кроме того, пусть $k=\max \left\{t: x_{t} \mid b\right\}$; понятно, что $k>l$. Если $a_{l+1}=\cdots=a_{n}=0$, то $a / x_{l} \geqslant b / x_{k}$, а если $\exists r>l$ такое, что $a_{r}>0$, то $a / x_{r}>b / x_{k}$; в обоих случаях $a \in \Delta^{R}(M)$ - противоречие.

Лемма 2.2. Следующие условия равносильны:

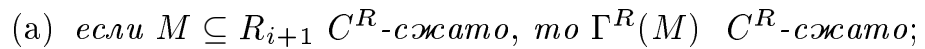

(b) если $a \in R_{i+1}, b \in R_{i}, b x_{n}<a$ и из $b x_{j}<$ а следует, ито $b x_{j} \in I_{i+1}$, mo $\exists l$ maкое, ито $a=b x_{l}$.

ДокАЗАТЕльСтво. Предположим, что выполнено (а), и пусть $a=x_{1}^{a_{1}} \cdots x_{n}^{a_{n}} \in$ $R_{i+1}, b=x_{1}^{b_{1}} \cdots x_{n}^{b_{n}} \in R_{i}$ такие, что $b x_{n}<a$ и из $b x_{j}<a$ следует, что $b x_{j} \in I_{i+1}$. Обозначим $M=\left\{m \in R_{i+1}: m \leqslant a\right\}$. Так как $M-C^{R}$-сжатое множество, то $\Gamma^{R}(M) C^{R}$-сжато. Аналогично доказательству леммы 2.1 получаем, что $\exists k$ такое, что $a / x_{k} \geqslant b$. Если $a / x_{k}>b$, то $b \notin \Gamma^{R}(M)$ (в силу свойств мономов $a$ и $b$ ), что противоречит $C^{R}$-сжатости множества $\Gamma^{R}(M)$.

Пусть теперь выполнено (b), но существует $C^{R}$-сжатое множество $M \subseteq R_{i+1}$ такое, что $\Gamma^{R}(M)$ не $C^{R}$-сжато. Возьмем в качестве $a$ максимальньй элемент в $M$. Снова аналогично доказательству леммы $2.1 \exists k$ такое, что $a / x_{k}-$ максимальный элемент в $\Gamma^{R}(M)$. Так как $\Gamma^{R}(M)$ не $C^{R}$-сжато, то $\exists b \in R_{i} \backslash \Gamma^{R}(M), b<a / x_{k}$. Элементы $a$ и $b$ удовлетворяют условию (b): в силу $b<a / x_{k}$ имеем $b x_{n}<a$; если $b x_{j}<a$, то $b x_{j} \in I_{i+1}$, так как иначе $b \in \Gamma^{R}(M)$. Поэтому из условия (b) вытекает, что $a=b x_{l}$ - противоречие.

Лемма 2.3. Если $M \subseteq R_{i}, A=R_{i-1} \backslash \Gamma^{R}(M), B=R_{i+1} \backslash \Delta^{R}(M), m o$

(a) $M \cap \Delta^{R}(A)=\varnothing$,

(b) $M \cap \Gamma^{R}(B)=\varnothing$.

ДокАЗАтЕльство. (а) Если $m \in M$, то $\Gamma^{R}(m) \subseteq R_{i-1} \backslash A$, а если $m \in \Delta^{R}(A)$, то $\exists i$ такое, что $m / x_{i} \in A$; поэтому если $m \in \Delta^{R}(A) \cap M$, то $A \cap \Gamma^{R}(M) \neq \varnothing-$ противоречие.

(b) Если $m \in M$, то $\Delta^{R}(m) \subseteq R_{i+1} \backslash B$, а если $m \in \Gamma^{R}(B)$, то $\exists i$ такое, что $m x_{i} \in B$; поэтому если $m \in \Gamma^{R}(B) \cap M$, то $B \cap \Delta^{R}(M) \neq \varnothing$ - противоречие.

Лемма 2.4. Пусть в кольце $R$ выполняется теорема Маколея, $M \subseteq R_{i}-$ $C^{R}$-сжатое множество, тогда $\Gamma^{R}(M) C^{R}$-сжато.

ДокАЗАТЕльство. Предположим, что сушествует $C^{R}$-сжатое множество $M \subseteq R_{i}$ такое, что $\Gamma^{R}(M)$ не $C^{R}$-сжато. Обозначим

$$
A=R_{i-1} \backslash \Gamma^{R}(M), \quad B=R_{i-1} \backslash C^{R}\left(\Gamma^{R}(M)\right) .
$$


В силу леммы 2.3 имеем, что $\Delta^{R}(A) \cap M=\varnothing$; следовательно, используя лемму 2.1, $C^{R}$-сжатость множества $M$ и теорему Маколея в кольце $R\left(B=D^{R} A\right)$, получаем, что $\Delta^{R}(B) \cap M=\varnothing$. $\mathrm{C}$ другой стороны, так как $\Gamma^{R}(M)$ не $C^{R}$-сжато, то $B \cap \Gamma^{R}(M) \neq \varnothing$, поэтому $\Delta^{R}(B) \cap M \neq \varnothing-$ противоречие.

СлЕДСТВИЕ 2.5. Если в кольие $R=A / I$ выполняется теорема Маколея, то идеал I удовлетворяет условию (b) леммы 2.2, в частности, если существует $a \in R_{i}$ такой, что ах $x_{j} \in I \forall j$, mо $\forall b \in A_{i+1}, b \geqslant a x_{n}$, имеем $b \in I_{i+1}$.

Однако условие $C^{R}$-сжатости $\Gamma^{R}$-образа любого $C^{R}$-сжатого множества не является достаточньм для выполнения теоремы Маколея, что видно на следующем примере.

Пример 2.6. $A=k\left[x_{1}, x_{2}, x_{3}\right], I$ порожден $x_{2} x_{3}$ и всеми мономами степени 3 .

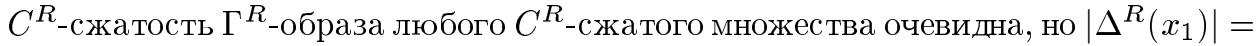
$3>\left|\Delta^{R}\left(x_{2}\right)\right|=2$.

Теорема 2.7. Для выполнения теоремы Маколея (1) в кольце $R$ необходимо и достаточно, чтобъ

$$
\Gamma^{R}\left(C^{R} M\right) \subseteq C^{R}\left(\Gamma^{R}(M)\right) \quad \forall i \geqslant 2, \quad \forall M \subseteq R_{i}
$$

ДокАЗАтЕЛьство. Пусть выполняется (2), но (1) неверно, т.е.

$$
\exists M \subseteq R_{i} \text { такое, что }\left|\Delta^{R}\left(D^{R} M\right)\right|>\left|\Delta^{R}(M)\right| .
$$

Обозначим

$$
\begin{aligned}
A & =\Delta^{R}\left(D^{R} M\right), \quad B=\Delta^{R}(M), \\
A^{\prime} & =R_{i+1} \backslash A, \quad B^{\prime}=R_{i+1} \backslash B .
\end{aligned}
$$

Так как $|A|>|B|$, то $\left|A^{\prime}\right|<\left|B^{\prime}\right|=\left|C^{R} B^{\prime}\right|$, кроме того, в силу леммы 2.1 множество $A D^{R}$-сжато, поэтому множество $A^{\prime} C^{R}$-сжато; следовательно, $A^{\prime} \subset$ $C^{R} B^{\prime}$ (включение строгое), из этого получаем, что $C^{R} B^{\prime} \cap A \neq \varnothing$ и $\Gamma^{R}\left(C^{R} B^{\prime}\right) \cap$ $D^{R} M \neq \varnothing$.

$\mathrm{C}$ другой стороны, по лемме 2.3 имеем, что $\Gamma^{R}\left(B^{\prime}\right) \cap M=\varnothing$, таким образом, $\left|\Gamma^{R}\left(B^{\prime}\right)\right|+|M| \leqslant\left|R_{i}\right|$, следовательно, $C^{R}\left(\Gamma^{R}\left(B^{\prime}\right)\right) \cap D^{R} M=\varnothing ;$ кроме того, из (2) следует, что $\Gamma^{R}\left(C^{R} B^{\prime}\right) \subseteq C^{R}\left(\Gamma^{R}\left(B^{\prime}\right)\right)$, т.е. $\Gamma^{R}\left(C^{R} B^{\prime}\right) \cap D^{R} M=\varnothing-$ противоречие.

Пусть теперь выполняется (1), но (2) неверно, т.е.

$$
\exists M \subseteq R_{i} \text { такое, что } \Gamma^{R}\left(C^{R} M\right) \nsubseteq C^{R}\left(\Gamma^{R}(M)\right) .
$$

В силу леммы 2.4 получаем, что $\left|\Gamma^{R}\left(C^{R} M\right)\right|>\left|\Gamma^{R}(M)\right|$.

Обозначим

$$
\begin{aligned}
A & =\Gamma^{R}\left(C^{R} M\right), \quad B=\Gamma^{R}(M), \\
A^{\prime} & =R_{i-1} \backslash A, \quad B^{\prime}=R_{i-1} \backslash B .
\end{aligned}
$$


Так как $|A|>|B|$, то $\left|A^{\prime}\right|<\left|B^{\prime}\right|=\left|D^{R} B^{\prime}\right|$, кроме того, в силу леммы 2.4 множество $A C^{R}$-сжато, поэтому множество $A^{\prime} D^{R}$-сжато, следовательно, $A^{\prime} \subset$ $D^{R} B^{\prime}$ (включение строгое); из этого получаем, что $D^{R} B^{\prime} \cap A \neq \varnothing_{\text {и }} \Delta^{R}\left(D^{R} B^{\prime}\right) \cap$ $C^{R} M \neq \varnothing$.

$\mathrm{C}$ другой стороны, по лемме 2.3 имеем, что $\Delta^{R}\left(B^{\prime}\right) \cap M=\varnothing$, таким образом, $\left|\Delta^{R}\left(B^{\prime}\right)\right|+|M| \leqslant\left|R_{i}\right|$, следовательно, $D^{R}\left(\Delta^{R}\left(B^{\prime}\right)\right) \cap C^{R} M=\varnothing ;$ кроме того, из (1) и леммы 2.1 следует, что $\Delta^{R}\left(D^{R} B^{\prime}\right) \subseteq D^{R}\left(\Delta^{R}\left(B^{\prime}\right)\right)$, т.е. $\Delta^{R}\left(D^{R} B^{\prime}\right) \cap C^{R} M=\varnothing$ - противоречие.

Из леммы 2.1 следует, что (1) равносильно

$$
\Delta^{R}\left(D^{R} M\right) \subseteq \Delta^{R}(M) \forall i \geqslant 1, \forall M \subseteq R_{i},
$$

однако (2) нельзя заменить на

$$
\left|\Gamma^{R}\left(C^{R} M\right)\right| \leqslant\left|C^{R}\left(\Gamma^{R}(M)\right)\right| \forall i \geqslant 2, \forall M \subseteq R_{i},
$$

что видно на следующем примере.

ПримеР 2.8. $A=k\left[x_{1}, x_{2}\right], I=\left(x_{1}^{3}, x_{1} x_{2}^{2}, x_{2}^{3}\right) A$. Очевидно, что

$$
\left|\Gamma^{R}\left(C^{R} M\right)\right| \leqslant\left|C^{R}\left(\Gamma^{R}(M)\right)\right| \forall i \geqslant 2, \forall M \subseteq R_{i}
$$

но теорема Маколея (2) не выполняется $\left(M=\left\{x_{1}^{2} x_{2}\right\}\right)$.

ТЕОрема 2.9. Если идеал $I$ таков, что множества $I_{i} D^{A}$-сжаты для всех $i \geqslant 1$ (лекс-сегментный идеал), то в $R=A / I$ выполняется теорема Маколея.

ДокаЗАТЕЛьство. Пусть $M$ - произвольное мономиальное множество в $R_{i}$. В силу теоремы Маколея в кольце $A$ имеем, что $\left|\Delta^{A}\left(M \cup I_{i}\right)\right| \geqslant\left|\Delta^{A}\left(D^{A}\left(M \cup I_{i}\right)\right)\right|$, а так как $D^{A}\left(M \cup I_{i}\right)=I_{i} \cup D^{R} M$, то $\Delta^{A}\left(M \cup I_{i}\right)=\Delta^{A}\left(I_{i}\right) \cup \Delta^{R}(M)$ и $\Delta^{A}\left(D^{A}\left(M \cup I_{i}\right)\right)=\Delta^{A}\left(I_{i}\right) \cup \Delta^{R}\left(D^{R} M\right)$. Поэтому $\left|\Delta^{A}\left(I_{i}\right)\right|+\left|\Delta^{R}(M)\right| \geqslant$ $\left|\Delta^{A}\left(I_{i}\right)\right|+\left|\Delta^{R}\left(D^{R} M\right)\right|$, т.е. $\left|\Delta^{R}(M)\right| \geqslant\left|\Delta^{R}\left(D^{R} M\right)\right|$.

Приведем еще одно условие, необходимое для выполнения теоремы Маколея, которое будет использовано в дальнейшем.

ТЕОрема 2.10. Если в $R=A / I$ выполняется теорема Маколея и $d-$ минимальная степень порожсдающих идеала $I$, то $\exists i \geqslant 1$ такое, что $x_{1}^{d-1+j} x_{i} \in I$ $\forall j \geqslant 0$.

ДоказАтельство. Достаточно доказать, что $\exists i \geqslant 1$ такое, что $x_{1}^{d-1} x_{i} \in I$.

Если $d=1$, то теорема очевидна. Пусть $d \geqslant 2$ и $x_{1}^{d-1} x_{i} \notin I_{d} \forall i \geqslant 1$, тогда $\Delta^{R}\left(x_{1}^{d-1}\right)=n$; но если $a \in I_{d}, x_{j} \mid a$, то $\Delta^{R}\left(a / x_{j}\right) \leqslant n-1-$ противоречие. 


\section{§ 3. Теорема Маколея для факторкольца по идеалу, порож,денному мономами от первых двух переменных}

Пусть $M \subseteq R_{v}$, положим

$$
M_{i=d}=\left\{m=x_{1}^{a_{1}} \cdots x_{n}^{a_{n}} \in M: a_{i}=d\right\} .
$$

Обозначим через $C_{i=d}^{R} M$ меньшие $\left|M_{i=d}\right|$ элементов в $\left(R_{v}\right)_{i=d}$. Будем говорить, что множество $M$ сжисато по индексу $i$ или $i$-сжато, если $C_{i=d}^{R} M=M_{i=d} \forall d$, $0 \leqslant d \leqslant v$.

Пусть $M_{v} \subseteq R_{v}$. Рассмотрим множества $M_{v}^{j}, j \geqslant 1$, определенные следующим образом:

$$
M_{v}^{1}=M_{v} ; \quad M_{v}^{j+1}=\bigcup_{d=0}^{v} C_{i=d}^{R} M_{v}^{j}, \quad i \equiv j(\bmod n), \quad 1 \leqslant i \leqslant n .
$$

Теорема 3.1. Пусть $I$ - идеал в $A$, порожденный мономами от переменных $x_{1}, x_{2}, R=A / I$. Если в $R^{\prime}=R /\left(x_{3}, x_{4}, \ldots, x_{n}\right)$ выполняется теорема Маколея, то в $R$ тоже выполняется теорема Маколея.

В силу теоремы 2.7 будем доказывать теорему Маколея в форме (2).

ДОКАЗАТЕЛЬСТВО ТЕОРЕМЫ 3.1 проведем индукцией по числу переменных в кольце $A$. База индукции при $n=2$ обеспечивается условием теоремы $\left(R^{\prime}=R\right.$ при $n=2$ ). Далее будем предполагать, что $n \geqslant 3$. Докажем сначала несколько вспомогательных лемм.

ЛЕмма 3.2. Если $M_{v} \subseteq R_{v}$, то $\exists p \geqslant 1$ такое, что $M_{v}^{p} i$-сжсато для $i=1,2, \ldots, n$.

ДокАЗАтЕльство. Перенумеруем элементы $R_{v}$ в соответствии с лексикографическим порядком, начиная с меншшего. Пусть $n(a)$ - номер элемента $a \in R_{v}$ и $n\left(M_{v}\right)$ - сумма номеров элементов $M_{v}$.

Так как $n\left(M_{v}^{i}\right) \geqslant 0$ и $n\left(M_{v}^{1}\right) \geqslant n\left(M_{v}^{2}\right) \geqslant \cdots \geqslant n\left(M_{v}^{i}\right) \geqslant \cdots$, то $\exists p$ такое, что $n\left(M_{v}^{p}\right)=n\left(M_{v}^{p+1}\right)=\cdots$, но тогда $M_{v}^{p}=M_{v}^{p+1}=\cdots$, а это означает, что $M_{v}^{p}$ $i$-сжато для $i=1,2, \ldots, n$.

ЛЕмма 3.3. Пусть теорема 3.1 верна при $n=k-1$, и пусть множества $M_{v} \subseteq R_{v}$ u $M_{v-1} \subseteq R_{v-1}$ такови, ито $\Gamma^{R}\left(M_{v}\right) \subseteq M_{v-1} n p u n=k$. Тогда $\Gamma^{R}\left(M_{v}^{j}\right) \subseteq M_{v-1}^{j} \forall j \geqslant 1$ npu $n=k$.

ДокАЗАТЕЛЬСТво. Проведем индукцию по $j$.

При $j=1$ утверждение следует из того, что $\Gamma^{R}\left(M_{v}\right) \subseteq M_{v-1}$. Пусть лемма доказана для $j$, докажем для $j+1$. Имеем:

$$
\begin{aligned}
\Gamma^{R}\left(M_{v}^{j+1}\right) & =\Gamma^{R}\left(\bigcup_{d=0}^{v} C_{i=d}^{R} M_{v}^{j}\right)=\bigcup_{d=0}^{v} \Gamma^{R}\left(C_{i=d}^{R} M_{v}^{j}\right) \\
& =\bigcup_{d=0}^{v}\left[\left(\Gamma^{R}\left[C_{i=d}^{R} M_{v}^{j}\right]\right)_{i=d} \cup\left(\Gamma^{R}\left[C_{i=d}^{R} M_{v}^{j}\right]\right)_{i=d-1}\right] \\
& =\left[\bigcup_{d=0}^{v}\left(\Gamma^{R}\left[C_{i=d}^{R} M_{v}^{j}\right]\right)_{i=d}\right] \cup\left[\bigcup_{d=1}^{v}\left(\Gamma^{R}\left[C_{i=d}^{R} M_{v}^{j}\right]\right)_{i=d-1}\right],
\end{aligned}
$$

где $i \equiv j(\bmod n), 1 \leqslant i \leqslant n$. 
В первой части объединения (3) $i$-й показатель остается постоянным под действием $\Gamma^{R}$. Если $i \geqslant 3$, то по условию леммы (теорема верна при $n=k-1$ ) имеем:

$$
\bigcup_{d=0}^{v}\left(\Gamma^{R}\left[C_{i=d}^{R} M_{v}^{j}\right]\right)_{i=d} \subseteq \bigcup_{d=0}^{v} C_{i=d}^{R}\left(\Gamma^{R}\left[\left(M_{v}^{j}\right)_{i=d}\right]\right) \subseteq \bigcup_{d=0}^{v} C_{i=d}^{R}\left(\Gamma^{R}\left[M_{v}^{j}\right]\right)
$$

Если $i<3$, то, применяя теорему 2.9 в кольце $k\left[x_{1}, x_{3}, \ldots, x_{n}\right]$ для идеала $I^{\prime}=$ $\left(x_{1}^{a_{1}}\right)$ или в кольце $k\left[x_{2}, x_{3}, \ldots, x_{n}\right]$ для идеала $I^{\prime}=\left(x_{2}^{a_{2}}\right)$, получаем, что

$$
\bigcup_{d=0}^{v}\left(\Gamma^{R}\left[C_{i=d}^{R} M_{v}^{j}\right]\right)_{i=d} \subseteq \bigcup_{d=0}^{v} C_{i=d}^{R}\left(\Gamma^{R}\left[\left(M_{v}^{j}\right)_{i=d}\right]\right) \subseteq \bigcup_{d=0}^{v} C_{i=d}^{R}\left(\Gamma^{R}\left[M_{v}^{j}\right]\right)
$$

Во второй части объединения (3) все показатели, кроме $i$-го, остаются постоянными. Пусть $a=x_{1}^{a_{1}} x_{2}^{a_{2}} \cdots x_{i}^{d} \cdots x_{n}^{a_{n}}$ - наибольший элемент в $C_{i=d}^{R} M_{v}^{j}$, тогда $a^{\prime}=x_{1}^{a_{1}} x_{2}^{a_{2}} \cdots x_{i}^{d-1} \cdots x_{n}^{a_{n}}$ - наибольший элемент в $\left(\Gamma^{R}\left[C_{i=d^{2}}^{R} M_{v}^{j}\right]\right)_{i=d-1}$. Пусть $b^{\prime}=x_{1}^{b_{1}} x_{2}^{b_{2}} \cdots x_{i}^{d-1} \cdots x_{n}^{b_{n}}<a^{\prime}, b^{\prime} \in\left(R_{v}\right)_{i=d-1}$, тогда покажем, что $b=x_{1}^{b_{1}} x_{2}^{b_{2}} \cdots x_{i}^{d} \cdots x_{n}^{a_{n}} \in\left(R_{v}\right)_{i=d}$ и $b<a$.

Если $i>2$, то $b \in\left(R_{v}\right)_{i=d}$, так как $I$ порожден мономами только от переменных $x_{1}, x_{2}$.

Если $i=1$, то $a_{1}=b_{1}+1=d$ и $a_{2} \geqslant b_{2}\left(a^{\prime}>b^{\prime}\right)$, следовательно, $b \in\left(R_{v}\right)_{i=d}$.

Если $i=2$, то аналогично $a_{2}=b_{2}+1=d$ и $a_{1} \geqslant b_{1}\left(a^{\prime}>b^{\prime}\right)$, следовательно, $b \in\left(R_{v}\right)_{i=d}$.

Кроме того, так как $b^{\prime}<a^{\prime}$, то $b=b^{\prime} x_{i}<a^{\prime} x_{i}=a$.

Таким образом, $b \in C_{i=d}^{R} M_{v}^{j}$ и $b^{\prime} \in\left(\Gamma^{R}\left[C_{i=d}^{R} M_{v}^{j}\right]\right)_{i=d-1}$, кроме того,

$$
\left|\left(\Gamma^{R}\left[C_{i=d}^{R} M_{v}^{j}\right]\right)_{i=d-1}\right|=\left|C_{i=d-1}^{R}\left(\Gamma^{R}\left[\left(M_{v}^{j}\right)_{i=d}\right]\right)\right|,
$$

поэтому

$$
\left(\Gamma^{R}\left[C_{i=d}^{R} M_{v}^{j}\right]\right)_{i=d-1}=C_{i=d-1}^{R}\left(\Gamma^{R}\left[\left(M_{v}^{j}\right)_{i=d}\right]\right)
$$

и, следовательно,

$$
\bigcup_{d=1}^{v}\left(\Gamma^{R}\left[C_{i=d}^{R} M_{v}^{j}\right]\right)_{i=d-1} \subseteq \bigcup_{d=1}^{v} C_{i=d-1}^{R}\left(\Gamma^{R}\left[\left(M_{v}^{j}\right)_{i=d}\right]\right) \subseteq \bigcup_{d=0}^{v-1} C_{i=d}^{R}\left[\Gamma^{R}\left(M_{v}^{j}\right)\right]
$$

Таким образом, имеем:

$$
\begin{aligned}
\Gamma^{R}\left(M_{v}^{j+1}\right) & \subseteq\left[\bigcup_{d=0}^{v} C_{i=d}^{R}\left(\Gamma^{R}\left[M_{v}^{j}\right]\right)\right] \cup\left[\bigcup_{d=0}^{v-1} C_{i=d}^{R}\left(\Gamma^{R}\left[M_{v}^{j}\right]\right)\right] \\
& =\bigcup_{d=0}^{v} C_{i=d}^{R}\left(\Gamma^{R}\left[M_{v}^{j}\right]\right) \subseteq \bigcup_{d=0}^{v} C_{i=d}^{R} M_{v-1}^{j}=M_{v-1}^{j+1}
\end{aligned}
$$

(последнее включение имеет место по предположению индукции).

ЛЕмма 3.4. Пусть $g=x_{1}^{g_{1}} x_{2}^{g_{2}} \cdots x_{n}^{g_{n}}, h=x_{1}^{h_{1}} x_{2}^{h_{2}} \cdots x_{n}^{h_{n}}, g, h \in R_{v}, g<h$. Ecли $h_{n}=0, h \in S \subseteq R_{v}$ u $S$ i-cжсато для $i=1,2, n$, mо $g \in S$. 
ДоКАЗАТЕЛЬСтво. Рассмотрим следующие случаи.

1) $g_{1}=h_{1}$ либо $g_{n}=h_{n}$. Утверждение леммы очевидно, так как $S 1$ и и $n$-сжато.

2) $g_{1}<h_{1}, g_{n}>h_{n}$ и $h_{2}=h_{3}=\cdots=h_{n-1}=0$. Рассмотрим моном $u=x_{1}^{u_{1}} x_{2}^{u_{2}} \cdots x_{n}^{u_{n}}$, где $u_{1}=h_{1}-g_{n}+h_{n}, u_{2}=\cdots=u_{n-1}=0, u_{n}=g_{n}$; $u \in R_{v}$, так как $u_{1}<h_{1}, u_{2}=h_{2}$, и $u \in S$, так как $u_{2}=h_{2}$ и $S$ 2-сжато. Кроме того, $h>u \geqslant g$ и $u_{n}=g_{n}$, следовательно, рассуждая аналогично случаю 1$)$, получаем, что $g \in S$.

3) $g_{1}<h_{1}, g_{n}>h_{n}$ и $\exists i, 2 \leqslant i \leqslant n-1$, такое, что $h_{i}>0$. Рассмотрим моном $u=x_{1}^{u_{1}} x_{2}^{u_{2}} \cdots x_{n}^{u_{n}}$, где $u_{1}=h_{1}, u_{n}=\min \left(\sum_{j=2}^{n-1} h_{j}, g_{n}\right), u_{n-1}=\sum_{j=2}^{n-1} h_{j}-u_{n}$, а остальные $u_{k}=0$. Моном $u \in R_{v}$, так как $u_{1}=h_{1}, u_{2} \leqslant h_{2}$, и $u \in S$, так как $u_{1}=h_{1}$ и $S$ 1-сжато. Кроме того, $h>u \geqslant g$, и если $u_{n}=g_{n}$, то, рассуждая аналогично случаю 1 ), получаем, что $g \in S$, а если $u_{n}<g_{n}$, то $u_{2}=\cdots=u_{n-1}=0$ и для окончания доказательства достаточно воспользоваться рассуждениями из случая 2).

ОКОНЧАНИЕ ДОКАЗАТЕЛЬСТВА ТЕОРЕМЫ 3.1. Пусть теорема доказана при $n-1$, докажем при $n$. Пусть $M_{v}$ - произвольное множество в $R_{v}$. Рассмотрим множества $M_{v}^{j}, j=1,2, \ldots$ По лемме $3.2 \exists p \geqslant 1$ такое, что $M_{v}^{p} i$-сжато для $i=1,2, \ldots, n$. Положим $S=M_{v}^{p}$ и $T=\left(\Gamma^{R}\left[M_{v}\right]\right)^{p}$. Применяя лемму 3.3 к $M_{v}$ и $\Gamma^{R}\left(M_{v}\right)$, получаем, что $\Gamma^{R}(S) \subseteq T$.

Если $S=R_{v}$, то $M_{v}=R_{v}$ и, очевидно, $\Gamma^{R}\left(C^{R} M_{v}\right)=C^{R}\left(\Gamma^{R}\left(M_{v}\right)\right)$.

Если $S \neq R_{v}$, то обозначим через $h=x_{1}^{h_{1}} x_{2}^{h_{2}} \cdots x_{n}^{h_{n}}$ наибольший моном в $S$ и через $g=x_{1}^{g_{1}} x_{2}^{g_{2}} \cdots x_{n}^{g_{n}}$ наименьший моном в $R_{v} \backslash S$. Рассмотрим два случая.

1) $g<h$. По лемме $3.4 h_{n}>0$, так как $S i$-сжато для $i=1,2, \ldots, n$. Положим $h^{\prime}=x_{1}^{h_{1}} x_{2}^{h_{2}} \cdots x_{n}^{h_{n}-1}$ и $g^{\prime}=x_{1}^{g_{1}} x_{2}^{g_{2}} \cdots x_{n}^{g_{n}-1}$, если $g_{n}>0$. Так как $\Gamma^{R}(S) \subseteq T$, то $h^{\prime} \in T$. Обозначим:

$$
S^{\prime}=(S \backslash\{h\}) \cup\{g\}, \quad T^{\prime}= \begin{cases}T, & g_{n}=0 \\ \left(T \backslash\left\{h^{\prime}\right\}\right) \cup\left\{g^{\prime}\right\}, & g_{n}>0\end{cases}
$$

Покажем, что $\Gamma^{R}\left(S^{\prime}\right) \subseteq T^{\prime}$.

Пусть $x \in S \backslash\{h\}$, тогда $h^{\prime} \notin \Gamma^{R}(x)$, следовательно, $\Gamma^{R}(S \backslash\{h\}) \subseteq T^{\prime}$.

Пусть $g_{n}>0$, тогда $g^{\prime} \in T^{\prime}$ по построению. Если $\exists i, 1 \leqslant i \leqslant n-1$, такое, что $g_{i}>0$, то $x_{1}^{g_{1}} x_{2}^{g_{2}} \cdots x_{i}^{g_{i}-1} \cdots x_{n}^{g_{n}} \in \Gamma^{R}(g)$. Моном $g^{\prime \prime}=x_{1}^{g_{1}} x_{2}^{g_{2}} \cdots x_{i}^{g_{i}-1} \cdots x_{n}^{g_{n}+1} \in$ $S$, так как $g^{\prime \prime}<g$ и $g$ - наименьший в $R_{v} \backslash S\left(g^{\prime \prime} \in R_{v}\right.$, так как $\left.n \geqslant 3\right)$. Таким образом, $\Gamma^{R}(g) \subseteq T \cup\left\{g^{\prime}\right\}$. Заметим далее, что $h_{1}>g_{1}(S i$-сжато) и, значит, $x_{1}^{g_{1}} x_{2}^{g_{2}} \cdots x_{i}^{g_{i}-1} \cdots x_{n}^{g_{n}}<h^{\prime}$, т.е. $\Gamma^{R}(g) \subseteq T^{\prime}$.

Пусть $g_{n}=0$, тогда $g^{\prime \prime} \in \Gamma^{R}\left(x_{1}^{g_{1}} x_{2}^{g_{2}} \cdots x_{i}^{g_{i}-1} \cdots x_{n}^{g_{n}+1}\right), g^{\prime \prime}<g$ и снова $\Gamma^{R}(g) \subseteq$ $T^{\prime}\left(g^{\prime \prime} \in R_{v}\right.$, так как $\left.n \geqslant 3\right)$.

Таким образом, $\Gamma^{R}\left(S^{\prime}\right) \subseteq T^{\prime}$.

Очевидно, что $S^{\prime} i$-сжато для $i=1,2, \ldots, n$.

После конечного числа применений операции ' получим $S^{\prime \cdots \prime}=C^{R} M_{v}$ и $\Gamma^{R}\left(S^{\prime \cdots \prime}\right)=\Gamma^{R}\left(C^{R} M_{v}\right) \subseteq T^{\prime \cdots \prime}=U$, следовательно, $\left|\Gamma^{R}\left(C^{R} M_{v}\right)\right| \leqslant|U|=$

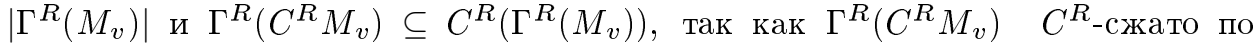
лемме $2.2(n \geqslant 3)$.

2) $g>h$. Значит, $S=C^{R} M_{v}$ и, следовательно, $\Gamma^{R}\left(C^{R} M_{v}\right)=C^{R}\left(\Gamma^{R}\left(M_{v}\right)\right)$.

Теорема 3.1 доказана полностью. 
Рассмотрим более подробно случай, когда $n=2$, т.е. $A=k\left[x_{1}, x_{2}\right]$.

Пусть $I_{v}=\left\{x_{1}^{l_{1}^{v}} x_{2}^{m_{1}^{v}}, x_{1}^{l_{2}^{v}} x_{2}^{m_{2}^{v}}, \ldots, x_{1}^{l_{s}^{v}} x_{2}^{m_{s}^{v}}\right\}$, причем $l_{1}^{v}>l_{2}^{v}>\cdots>l_{s}^{v}$. Составим векторы:

$$
\begin{aligned}
l^{v} & =\left(l_{0}^{v}, l_{1}^{v}, \ldots, l_{s+1}^{v}\right)=\left(v+1, l_{1}^{v}, l_{2}^{v}, \ldots, l_{s}^{v},-1\right), \\
\Delta l^{v} & =\left(\Delta l_{0}^{v}, \Delta l_{1}^{v}, \ldots, \Delta l_{s}^{v}\right) \\
& =\left(l_{0}^{v}-l_{1}^{v}-1, l_{1}^{v}-l_{2}^{v}-1, \ldots, l_{s-1}^{v}-l_{s}^{v}-1, l_{s}^{v}-l_{s+1}^{v}-1\right), \\
s^{v} & =\left(s_{1}^{v}, s_{2}^{v}, \ldots, s_{r}^{v}\right),
\end{aligned}
$$

где вектор $s^{v}$ получается из $\Delta l^{v}$ удалением компонент, равных нулю $\left(s^{v}\right.$ может быть пустым).

Множество вида $\left\{x_{1}^{a_{1}} x_{2}^{a_{2}}, x_{1}^{a_{1}-1} x_{2}^{a_{2}+1}, \ldots, x_{1}^{a_{1}-q} x_{2}^{a_{2}+q}\right\}$ будем называть отрезком.

Теорема 3.5. Теорема Маколея в кольие $R=A / I=k\left[x_{1}, x_{2}\right] / I$ выполняется тогда и только тогда, когда идеал I удовлетворяет следующим условиям:

(a) если $m \in R_{v}$, но $m x_{1} \in I, m x_{2} \in I$, mо $\forall m^{\prime} \in R_{v+1}, m^{\prime} \geqslant m x_{2}$, имеем $m^{\prime} \in I$

(b) $\forall v \geqslant 1$ :

(i) если $\Delta l_{0}^{v}=0, \Delta l_{s}^{v}=0$, mo $s_{i}^{v} \leqslant s_{i+1}^{v}$ nрu $i=1,2, \ldots, r-1$;

(ii) ecлu $\Delta l_{0}^{v}=0, \Delta l_{s}^{v}>0$, mo $s_{i}^{v} \leqslant s_{i+1}^{v}$ npu $i=1,2, \ldots, r-2$;

(iii) если $\Delta l_{0}^{v}>0, \Delta l_{s}^{v}=0$, mo $r=1, s_{1}^{v}=\Delta l_{0}^{v}=1$;

(iv) если $\Delta l_{0}^{v}>0, \Delta l_{s}^{v}>0$, mо либо $l^{v}=(v+1,-1)$, либо $r=2, s^{v}=$ $\left(\Delta l_{0}^{v}, \Delta l_{s}^{v}\right) u s_{1}^{v}=1$.

ДокАЗАТЕльство. Пусть в кольце $R$ выполняется теорема Маколея (например, в формулировке (2)), т.е.

$$
\Gamma^{R}\left(C^{R} M\right) \subseteq C^{R}\left(\Gamma^{R}(M)\right) \forall v \geqslant 2, \forall M \subseteq R_{v}
$$

Выполнение условия (а) вытекает из следствия 2.5.

Предположим теперь, что условие (b) не выполняется.

(i) Пусть $\exists v \geqslant 2$ такое, что $\Delta l_{0}^{v}=0, \Delta l_{s}^{v}=0$, но $\exists j, 1 \leqslant j \leqslant r-1$, такое, что $s_{j}^{v}>s_{j+1}^{v}$. Пусть $j$ - максимальное с таким условием, т.е. $s_{j}^{v}>s_{j+1}^{v} \leqslant s_{j+2}^{v} \leqslant$ $\cdots \leqslant s_{r}^{v}$. Пусть $s_{i}^{v}=l_{j_{i}}^{v}-l_{j_{i}+1}^{v}-1, i=1,2, \ldots, r$. Обозначим

$$
B_{i}=\left\{w \in F_{v}: x_{1}^{l_{j_{i}}^{v}} x_{2}^{m_{j_{i}}^{v}}>w>x_{1}^{l_{j_{i}+1}^{v}} x_{2}^{m_{j_{i}+1}^{v}}\right\}
$$

при $i=1,2, \ldots, r$. Очевидно, что $\left|B_{i}\right|=s_{i}^{v},\left|\Gamma^{R}\left(B_{i}\right)\right|=s_{i}^{v}+1$ при всех $i$. Положим

$$
M=B_{j} \cup \bigcup_{i=j+2}^{r} B_{i},
$$

тогда

$$
C^{R} M=D_{j} \cup \bigcup_{i=j+1}^{r} B_{i}
$$


где $D_{j} \subset B_{j},\left|D_{j}\right|=\left|B_{j}\right|-\left|B_{j+1}\right|>0$. Очевидно, что

$$
\begin{gathered}
\left|\Gamma^{R}(M)\right|=s_{j}^{v}+1+\sum_{i=j+2}^{r}\left(s_{i}^{v}+1\right), \\
\left|\Gamma^{R}\left(C^{R} M\right)\right|=\left|D_{j}\right|+1+\sum_{i=j+1}^{r}\left(s_{i}^{v}+1\right) .
\end{gathered}
$$

Таким образом, $\left|\Gamma^{R}(M)\right|=\left|\Gamma^{R}\left(C^{R} M\right)\right|-1$, поэтому $\Gamma^{R}\left(C^{R} M\right) \nsubseteq C^{R}\left(\Gamma^{R}(M)\right)-$ противоречие.

(ii) Пусть $\exists v \geqslant 2$ такое, что $\Delta l_{0}^{v}=0, \Delta l_{s}^{v}>0$, но $\exists j, 1 \leqslant j \leqslant r-2$, такое, что $s_{j}^{v}>s_{j+1}^{v}$. Будем считать, что $r \geqslant 3$, так как иначе условие очевидно. Пусть $j-$ максимальное с данным условием, т.е. $s_{j}^{v}>s_{j+1}^{v} \leqslant s_{j+2}^{v} \leqslant \cdots \leqslant s_{r-1}^{v}$. Используя обозначения, введенные выше, заметим, что $\left|B_{i}\right|=s_{i}^{v},\left|\Gamma^{R}\left(B_{i}\right)\right|=s_{i}^{v}+1$ при $i<r$ и $\left|B_{r}\right|=s_{r}^{v},\left|\Gamma^{R}\left(B_{r}\right)\right|=s_{r}^{v}$. Положим

$$
M=B_{j} \cup \bigcup_{i=j+2}^{r} B_{i},
$$

тогда

$$
C^{R} M=D_{j} \cup \bigcup_{i=j+1}^{r} B_{i}
$$

где $D_{j} \subset B_{j},\left|D_{j}\right|=\left|B_{j}\right|-\left|B_{j+1}\right|>0$. Очевидно, что

$$
\begin{gathered}
\left|\Gamma^{R}(M)\right|=s_{j}^{v}+\sum_{i=j+2}^{r}\left(s_{i}^{v}+1\right), \\
\left|\Gamma^{R}\left(C^{R} M\right)\right|=\left|D_{j}\right|+\sum_{i=j+1}^{r}\left(s_{i}^{v}+1\right) .
\end{gathered}
$$

Таким образом, $\left|\Gamma^{R}(M)\right|=\left|\Gamma^{R}\left(C^{R} M\right)\right|-1$, значит, $\Gamma^{R}\left(C^{R} M\right) \nsubseteq C^{R}\left(\Gamma^{R}(M)\right)-$ противоречие.

(iii) Пусть $\exists v \geqslant 2$ такое, что $\Delta l_{0}^{v}>0, \Delta l_{s}^{v}=0$, но либо $r>1$, либо $s_{1}^{v}>1$. Заметим сразу, что $I_{v} \neq \varnothing$, следовательно, в силу теоремы 2.10 неравенство $s_{1}^{v}>1$ невозможно. Поэтому остается лишь случай, когда $r>1$. Положим

$$
M=\left\{x_{1}^{v}\right\}
$$

Так как $r>1$, то $C^{R} M \neq M$, а так как $\Delta l_{s}^{v}=0$, то $\left|\Gamma^{R}\left(C^{R} M\right)\right|=2$, но $\left|\Gamma^{R}(M)\right|=1$, следовательно, $\Gamma^{R}\left(C^{R} M\right) \nsubseteq C^{R}\left(\Gamma^{R}(M)\right)$ - противоречие.

(iv) Пусть $\exists v \geqslant 2$ такое, что $\Delta l_{0}^{v}>0, \Delta l_{s}^{v}>0$ и либо $r=1$, но $l^{v} \neq(v+1,-1)$, либо $r=2$, но $s^{v} \neq\left(1, \Delta l_{s}^{v}\right)$, либо $r>2$.

Если $r=1$, то из условий $\Delta l_{0}^{v}>0, \Delta l_{s}^{v}>0$ получаем $l^{v}=(v+1,-1)$.

Если $r=2$, то $I_{v} \neq \varnothing$, следовательно, в силу теоремы 2.10 получаем, что $s_{1}^{v}=1$, т.е. $s^{v}=\left(1, \Delta l_{s}^{v}\right)$. 
Остается случай $r>2$. Положим (используя введенные выше обозначения)

$$
M=\left\{x_{1}^{v}\right\} \cup B_{r} .
$$

Так как $r>2$, то $C^{R} M \cap\left\{x_{1}^{v}\right\}=\varnothing$, следовательно,

$$
\left|\Gamma^{R}(M)\right|=\left|B_{r}\right|+1, \quad\left|\Gamma^{R}\left(C^{R} M\right)\right|=\left|B_{r}\right|+\left|\Gamma^{R}\left(C^{R} M \backslash B_{r}\right)\right|=\left|B_{r}\right|+2
$$

и в результате $\Gamma^{R}\left(C^{R} M\right) \nsubseteq C^{R}\left(\Gamma^{R}(M)\right)$ - противоречие.

Таким образом, условие (b) доказано.

Пусть теперь выполняются условия (a) и (b), докажем теорему Маколея. Покажем сначала, что

$$
\left|\Gamma^{R}\left(C^{R} M\right)\right| \leqslant\left|\Gamma^{R}(M)\right| \forall v \geqslant 2, \forall M \subseteq R_{v} .
$$

Для этого воспользуемся условием (b).

(i) $\Delta l_{0}^{v}=0, \Delta l_{s}^{v}=0$.

Положим $M^{i}=M \cap B_{i}$. Представим каждое из множеств $M^{i}$ в виде объединения максимальных отрезков:

$$
M^{i}=\bigcup_{j=1}^{r_{i}} B_{i j}
$$

причем $B_{i j} \cup B_{i k}$ не является отрезком при $j \neq k$.

Положим

$$
N(M)=\sum_{i=1}^{r} r_{i}
$$

Очевидно, что

$$
\left|\Gamma^{R}(M)\right|=\sum_{i=1}^{r} \sum_{j=1}^{r_{i}}\left(\left|B_{i j}\right|+1\right)=|M|+N(M) .
$$

В силу условия (b) имеем $s_{i}^{v} \leqslant s_{i+1}^{v}$ при $i=1,2, \ldots, r-1$, следовательно, $N(M) \geqslant N\left(C^{R} M\right)$, таким образом,

$$
\left|\Gamma^{R}(M)\right|=|M|+N(M) \geqslant\left|C^{R} M\right|+N\left(C^{R} M\right)=\left|\Gamma^{R}\left(C^{R} M\right)\right| .
$$

(ii) $\Delta l_{0}^{v}=0, \Delta l_{s}^{v}>0$.

Пусть $M^{i}, B_{i j}, N(M)$ - как и выше. Рассмотрим множества

$$
S=M \cap\left(\bigcup_{i=1}^{r-1} B_{i}\right), \quad S^{\prime} \subseteq \bigcup_{i=1}^{r-1} B_{i},
$$

причем $S^{\prime}$ состоит из меньших элементов в $\bigcup_{i=1}^{r-1} B_{i},\left|S^{\prime}\right|=|S|$. Тогда аналогично доказанному в предыдушем пункте получаем, что $\left|\Gamma^{R}(S)\right| \geqslant\left|\Gamma^{R}\left(S^{\prime}\right)\right|$ и, следовательно, $\left|\Gamma^{R}(M)\right| \geqslant\left|\Gamma^{R}\left(S^{\prime} \cup\left(M \cap B_{r}\right)\right)\right|$. Очевидно, что $N\left(S^{\prime}\right)+1 \geqslant N\left(C^{R} M\right)$, следовательно,

$$
\begin{aligned}
\left|\Gamma^{R}(M)\right| & =\left|\Gamma^{R}(S)\right|+\left|\Gamma^{R}(M \backslash S)\right| \geqslant\left|\Gamma^{R}\left(S^{\prime}\right)\right|+|M \backslash S|=\left|S^{\prime}\right|+N\left(S^{\prime}\right)+|M \backslash S| \\
& =|M|+N\left(S^{\prime}\right) \geqslant\left|C^{R} M\right|+N\left(C^{R} M\right)-1=\left|\Gamma^{R}\left(C^{R} M\right)\right| .
\end{aligned}
$$


(iii) $\Delta l_{0}^{v}>0, \Delta l_{s}^{v}=0$. Из условия (b) следует, что $\left|R_{v}\right|=1$ и, очевидно, $\left|\Gamma^{R}\left(C^{R} M\right)\right|=\left|\Gamma^{R}(M)\right|$.

(iv) $\Delta l_{0}^{v}>0, \Delta l_{s}^{v}>0$. Если $r=1$, то из условия (b) следует, что $l^{v}=(v+1,-1)$, т.е. в степени $v$ нет мономов, принадлежащих идеалу, следовательно, по теореме Маколея для кольца $A$ имеем $\left|\Gamma^{R}\left(C^{R} M\right)\right| \leqslant\left|\Gamma^{R}(M)\right|$. Если $r=2$, то из условия (b) следует, что $s^{v}=\left(1, \Delta l_{s}^{v}\right)$. Если $M=R_{v}$, то неравенство (4) очевидно. Пусть $M \neq R_{v}$, тогда $\left|\Gamma^{R}\left(C^{R} M\right)\right|=\left|C^{R} M\right|$ и

$$
\left|\Gamma^{R}(M)\right|=\left|\Gamma^{R}\left(M \cap B_{1}\right)\right|+\left|\Gamma^{R}\left(M \cap B_{2}\right)\right| \geqslant\left|M \cap B_{1}\right|+\left|M \cap B_{2}\right|=|M|,
$$

следовательно, $\left|\Gamma^{R}\left(C^{R} M\right)\right| \leqslant\left|\Gamma^{R}(M)\right|$.

Таким образом, (4) доказано.

Легко видеть, что при $n=2$ условие (а) теоремы эквивалентно условию (b) леммы 2.2 , поэтому из (а) следует, что если множество $M C^{R}$-сжато, то $\Gamma^{R}(M)$ тоже $C^{R}$-сжато.

Применяя (4) и пользуясь $C^{R}$-сжатостью множеств $C^{R}\left(\Gamma^{R}(M)\right)$ и $\Gamma^{R}\left(C^{R} M\right)$, получаем $\Gamma^{R}\left(C^{R} M\right) \subseteq C^{R}\left(\Gamma^{R}(M)\right)$.

Теорема 3.5 доказана полностью.

ЗАмЕчАнИЕ 3.6. Условие (а) теоремы 3.5 достаточно проверить при $v=1,2$, $\ldots, q-1$, а условие (b) - при $v=1,2, \ldots, 2 q$, где $q$ - максимальная степень порождающих идеала $I$.

СлЕДСТВИЕ 3.7. Пусть $I-$ идеал в $A=k\left[x_{1}, \ldots, x_{n}\right]$, порожденный мономами от переменных $x_{1}, x_{2}, R=A / I$. Следующие условия равносильны:

(a) идеал $I^{\prime}=I /\left(x_{3}, \ldots, x_{n}\right) \in k\left[x_{1}, x_{2}\right]$ удовлетворяет условиям (a) $u$ (b) теоремы 3.5 ;

(b) в кольие $k\left[x_{1}, x_{2}\right] / I^{\prime}$ вьлолняется теорема Маколея;

(c) теорема Маколея в кольце $R$ выполняется при всех $n \geqslant 2$.

Следует отметить, что теорема, обратная к теореме 3.1 , неверна:

Пример 3.8. Рассмотрим идеалы $I \subseteq k\left[x_{1}, x_{2}\right]$ и $I^{\prime} \subseteq k\left[x_{1}, x_{2}, x_{3}\right]$, порожденные мономами $x_{1}^{5}, x_{1}^{2} x_{2}^{5}, x_{2}^{7}, x_{1}^{4} x_{2}^{4}$. В кольце $R=k\left[x_{1}, x_{2}\right] / I$ теорема Маколея не вьполняется, так как при $v=7$ нарушается условие (i) теоремы 3.5. Докажем, что в кольце $R^{\prime}=k\left[x_{1}, x_{2}, x_{3}\right] / I^{\prime}$ теорема Маколея выполняется. Воспользуемся формулировкой (2). Если $i<7$, то теорема Маколея выполняется, так как $I^{\prime}$ в этих степенях совпадает с лекс-сегментньм идеалом. Если $i \geqslant 7$, то $R_{i}^{\prime}=x_{3}^{i-7} R_{7}^{\prime}$, поэтому достаточно рассмотреть случаи $i=7$ и $i=8$. Как было проверено на компьютере, в этих двух случаях теорема Маколея выполняется.

\section{§4. Теорема Маколея для факторколец} по лексическим и вполне лексическим идеалам

Если $u, v \in A_{i}, u \geqslant v$, то множество мономов $\left\{m \in A_{i}: u \geqslant m \geqslant v\right\}$ в $A$ будем назьвать отрезком и обозначать через $[u, v]$, кроме того, идеал, порожденньй $[u, v]$, будем назьвать лексическим и обозначать через $L[u, v]$. Однородный идеал $I$, у которого все $I_{j}$ являются отрезками, будем называть вполне лексическим.

В статье [3] было дано следующее описание вполне лексических идеалов: 
ТЕОрема 4.1. Пусть $u=x_{1}^{a_{1}} \cdots x_{n}^{a_{n}}, v=x_{1}^{b_{1}} \cdots x_{n}^{b_{n}} ; I=L[u, v]$ является вполне лексическим тогда и только тогда, когда выполнено одно из следующих условий:

(a) $v=x_{n}^{d}, u \geqslant x_{2}^{d}$;

(b) $v>x_{n}^{d}, a_{1}>0, a_{1}=b_{1}, u=x_{1}^{a_{1}} x_{2}^{d-a_{1}}, v=x_{1}^{a_{1}} x_{n}^{d-a_{1}}$;

(c) $v>x_{n}^{d}, a_{1}>0, a_{1}>b_{1} u \forall w<v, \operatorname{deg} w=d, \exists i>1$ maкое, ито $x_{i} \mid w u$ $w x_{1} / x_{i} \leqslant u$.

Для доказательства этой теоремы авторы статьи [3] использовали весьма сложную конструкцию минимальных резольвент из статьи [4], а также аппарат теории производных функторов. Ниже приводится более простое доказательство теоремы 4.1 без использования методов гомологической алгебры.

Если $m$ - моном, то положим $M(m)=\max \left\{k: x_{k} \mid m\right\}$.

ЛЕМма 4.2. Если $I=L[u, v], u, v$ удовлетворяют условию (а) теоремы 4.1, то $I_{d+1}$ - отрезок.

ДокАЗАТЕЛЬСТво. ПредПоложим, что $m \in A_{d+1} \backslash I_{d+1}, m<x_{1} u$.

Если $x_{1} \mid m$, то $m / x_{1}<u$, т.е. $m / x_{1} \in I_{d}-$ противоречие.

Если $x_{1} \nmid m$, то $m / x_{M(m)} \leqslant x_{2}^{d} \leqslant u$ - снова противоречие.

ЛЕМма 4.3. Если $I=L[u, v], u, v$ удовлетворяют условию (b) теоремы 4.1, то $I_{d+1}$ - отрезок.

ДОКАЗАТЕЛЬСТво. ПредПоложим, что $m \in\left[x_{1} u, x_{n} v\right] \backslash I_{d+1}$.

Если $x_{1}^{a_{1}+1} \mid m$, то $u>m / x_{1} \geqslant v$-противоречие.

Если $x_{1}^{a_{1}} \mid m$, но $x_{1}^{a_{1}+1} \nmid m$, то $u \geqslant m / x_{M(m)} \geqslant v$ - снова противоречие.

ЛЕмма 4.4. Если $I=L[u, v], u, v$ удовлетворяют условию (с) теоремъ 4.1, то $I_{d+1}$ - отрезок.

ДокАЗАТЕЛьство. Предположим, что $m=x_{1}^{c_{1}} \cdots x_{n}^{c_{n}} \in\left[x_{1} u, x_{n} v\right] \backslash I_{d+1}$.

Разберем следующие случаи:

1) $a_{1}>b_{1}+1$.

Если $c_{1}>b_{1}+1$, то $u>m / x_{1}>v-$ противоречие.

Если $c_{1} \leqslant b_{1}+1$, то $u>m / x_{M(m)} \geqslant v-$ противоречие.

2) $a_{1}=b_{1}+1$.

Если $c_{1}=a_{1}+1$, то $u>m / x_{1}>v-$ противоречие.

Если $c_{1}=b_{1}$, то $u>m / x_{M(m)} \geqslant v-$ противоречие.

Если $c_{1}=a_{1}$, то $u>m / x_{1}$. Если $m / x_{1} \geqslant v$, то $m / x_{1} \in I_{d}$ - противоречие, $\mathrm{a}$ если $m / x_{1}<v$, то в силу условия (c) $\exists i>1$ такое, что $x_{i} \mid\left(m / x_{1}\right)$ и $\frac{m}{x_{1}} \frac{x_{1}}{x_{i}} \leqslant u$, т.е. $u \geqslant m / x_{i}$. Кроме того, так как $c_{1}=a_{1}$, то $m / x_{i}>v$, следовательно, $m / x_{i} \in I_{d}-$ противоречие.

ЛЕмма 4.5. Если $I=L[u, v], u, v$ удовлетворяют условию (а) теоремъ 4.1, то $I$ - вполне лексический.

ДоКАЗАТЕЛЬСТво. В силу леммы $4.2 I_{d+1}$ - отрезок, $x_{1} u$ и $v x_{n}$ удовлетворяют условию (a) теоремы 4.1, следовательно, по лемме $4.2 I_{d+2}$ - отрезок и т. д. 
ЛЕмма 4.6. Если $I=L[u, v], u, v$ удовлетворяют условию (с) теоремы 4.1, то $I$ - вполне лексический.

ДокАЗАТЕЛЬСтво. Обозначим $u^{\prime}=x_{1} u, v^{\prime}=x_{n} v$. Очевидно, что $a_{1}^{\prime}>0$, $a_{1}^{\prime}>b_{1}^{\prime}$. Пусть $w=x_{1}^{c_{1}} \cdots x_{n}^{c_{n}} \in A_{d+1}, w<v^{\prime}$, тогда $M(w)>1$ и $u>w / x_{M(w)}$, так как $c_{1} \leqslant b_{1}^{\prime}=b_{1}<a_{1}$, следовательно, $u^{\prime}>w x_{1} / x_{M(w)}$, т.е. $u^{\prime}, v^{\prime}$ удовлетворяют условию (с) теоремы 4.1. Кроме того, по лемме $4.4 I_{d+1}$ - отрезок, поэтому, применяя лемму 4.4 к $I_{d+1}$, получаем, что $I_{d+2}-$ отрезок и т. д.

Лемма 4.7. Если $I=L[u, v], u, v$ удовлетворяют условию (b) теоремы 4.1, то $I$ - вполне лексический.

ДокАЗАтЕльство. Обозначим $u^{\prime}=x_{1} u, v^{\prime}=x_{n} v$. Очевидно, что $a_{1}^{\prime}>0$, $a_{1}^{\prime}>b_{1}^{\prime}$. Пусть $w=x_{1}^{c_{1}} \cdots x_{n}^{c_{n}} \in A_{d+1}, w<v^{\prime}=x_{1}^{a_{1}} x_{n}^{d-a_{1}+1}$, тогда $c_{1}<a_{1}$, следовательно, $w x_{1} / x_{M(w)}<u^{\prime}$, т.е. $u^{\prime}, v^{\prime}$ удовлетворяют условию (c) теоремы 4.1. Кроме того, по лемме $4.3 I_{d+1}$ - отрезок, поэтому, применяя лемму $4.6 \mathrm{k} I_{d+1}$, получаем, что $I$ - вполне лексический.

ЛЕмма 4.8. Если $I=L[u, v]$ u $I_{d+1}-$ отрезок, $m о$ $u \geqslant x_{2}^{d}$.

ДокАЗАтЕЛьство. Предположим, что $u<x_{2}^{d}$, тогда $u x_{1}>x_{2}^{d+1}>v x_{n}$, а так как $I_{d+1}$ - отрезок, то $x_{2}^{d+1} \in I_{d+1}$, следовательно, $x_{2}^{d} \in I_{d}$ - противоречие.

Лемма 4.9. Если $I=L[u, v], v>x_{n}^{d} u I_{d+1}-$ отрезок, то $a_{1}>0 u$ выполняется одно из следующих условий:

(a) $a_{1}=b_{1} u \quad u=x_{1}^{a_{1}} x_{2}^{d-a_{1}}, v=x_{1}^{a_{1}} x_{n}^{d-a_{1}}$;

(b) $a_{1}>b_{1} u \forall w<v, \operatorname{deg} w=d, \exists i>1$ maкое, что $x_{i} \mid w u w x_{1} / x_{i} \leqslant u$.

ДокАЗАТЕльство. Предположим сначала, что $a_{1}=0$, тогда $u x_{1}>x_{n}^{d} x_{1}>$ $v x_{n}$, поэтому либо $x_{n}^{d} \in I_{d}$, либо $x_{1} x_{n}^{d-1} \in I_{d}$ - в обоих случаях противоречие, т.е. $a_{1}>0$.

Пусть $a_{1}=b_{1}$. Если $v>x_{1}^{a_{1}} x_{n}^{d-a_{1}}$, то $u x_{1}>x_{1}^{a_{1}+1} x_{n}^{d-a_{1}}>v x_{n}$, следовательно, либо $x_{1}^{a_{1}} x_{n}^{d-a_{1}} \in I_{d}$, либо $x_{1}^{a_{1}+1} x_{n}^{d-a_{1}-1} \in I_{d}$-в обоих случаях противоречие, т.е. $v=x_{1}^{a_{1}} x_{n}^{d-a_{1}}$. Если $u<x_{1}^{a_{1}} x_{2}^{d-a_{1}}$, то $u x_{1}>x_{1}^{a_{1}} x_{2}^{d-a_{1}+1}>v x_{n}$, следовательно, либо $x_{1}^{a_{1}} x_{2}^{d-a_{1}} \in I_{d}$, либо $x_{1}^{a_{1}-1} x_{2}^{d-a_{1}+1} \in I_{d}$ - в обоих случаях противоречие, т.е. $u=x_{1}^{a_{1}} x_{2}^{d-a_{1}}$.

Пусть $a_{1}>b_{1}$, но $\exists w<v, \operatorname{deg} w=d$, такой, что $\forall i>1$ такого, что $x_{i} \mid w$, имеем $w x_{1} / x_{i}>u$. В этом случае $u x_{1}>w x_{1}>u x_{M(w)} \geqslant v x_{n}$, следовательно, $\exists j$ такое, что $w x_{1} / x_{j} \in[u, v]$, но если $j=1$, то получаем, что $w \in[u, v]$, а если $j>1$, то получаем, что $w x_{1} / x_{j} \in[u, v]$ - в обоих случаях противоречие.

ДОКАЗАТЕЛЬСТво ТЕОРЕМЫ 4.1. Если $I$ - вполне лексический, то, применяя леммы 4.8 (если $v=x_{n}^{d}$ ) и 4.9 (если $v>x_{n}^{d}$ ), получаем выполнение условий (a), (b) или (c). Если же выполняется одно из условий (a), (b) или (c), то, применяя леммы $4.5,4.7$ или 4.6 соответственно, получаем, что $I$ - вполне лексический.

ТЕОрема 4.10. Если идеал I порожсден мономами $x_{1}^{d-1} x_{i_{1}}, x_{1}^{d-1} x_{i_{2}}, \ldots$, $x_{1}^{d-1} x_{i_{s}}, i_{1}<i_{2}<\cdots<i_{s}$, то в $R=A / I$ выполняется теорема Маколея. 
ДокАЗАТЕльСтво. Нужно доказать, что

$$
\left|\Delta^{R}\left(D^{R} M\right)\right| \leqslant\left|\Delta^{R}(M)\right| \forall v \geqslant 1, \forall M \in R_{v} .
$$

Если $v=d+r, r \geqslant 0$, то $I_{d+r}=x_{1}^{d-1} \cdot\left\{x_{i_{1}}, x_{i_{2}}, \ldots, x_{i_{s}}\right\} \cdot A_{r}$ и $\left|I_{d+r}\right|=\left(\begin{array}{c}n+r \\ r+1\end{array}\right)-$ $\left(\begin{array}{c}n-s+r \\ r+1\end{array}\right)$. Следовательно, $D^{A}\left(I_{d+r}\right)=x_{1}^{d-1} \cdot\left\{x_{1}^{r+1}, x_{1}^{r} x_{2}, x_{1}^{r} x_{3}, \ldots, x_{s} x_{n}^{r}\right\}$ состоит из всех мономов степени $d+r$, бо́льших $x_{1}^{d-1} x_{s+1}^{r+1}$, так как $\left|D^{A}\left(I_{d+r}\right)\right|=$ $\left(\begin{array}{c}n+r \\ r+1\end{array}\right)-\left(\begin{array}{c}n-s+r \\ r+1\end{array}\right)$ и $D^{A}\left(I_{d+r}\right)$ состоит из старших мономов в $A_{d+r}$.

Пусть $M_{d+r}$ - произвольное мономиальное множество в $R_{d+r}$. Заметим, что $\left|\Delta^{R}\left(D^{R} M_{d+r}\right)\right|=\left|\Delta^{A}\left(D^{R} M_{d+r} \cup I_{d+r}\right)\right|-\left|\Delta^{A}\left(I_{d+r}\right)\right|$ и $\left|\Delta^{R}\left(M_{d+r}\right)\right|=$ $\left|\Delta^{A}\left(M_{d+r} \cup I_{d+r}\right)\right|-\left|\Delta^{A}\left(I_{d+r}\right)\right|$, кроме того, по теореме Маколея в кольце $A$ имеем, что $\left|\Delta^{A}\left(M_{d+r} \cup I_{d+r}\right)\right| \geqslant\left|\Delta^{A}\left(D^{A}\left(M_{d+r} \cup I_{d+r}\right)\right)\right|$. Таким образом, достаточно показать, что

$$
\left|\Delta^{A}\left(D^{R} M_{d+r} \cup I_{d+r}\right)\right|=\left|\Delta^{A}\left(D^{A}\left(M_{d+r} \cup I_{d+r}\right)\right)\right| .
$$

Пусть $m_{0}=x_{1}^{a_{1}} x_{2}^{a_{2}} \cdots x_{n}^{a_{n}}$ - минимальный элемент в $D^{R} M_{d+r}$. Если $m_{0}<$ $x_{1}^{d-1} x_{i_{s}} x_{n}^{r}$, то $D^{A}\left(M_{d+r} \cup I_{d+r}\right)=D^{R} M_{d+r} \cup I_{d+r}$ и (6) очевидно.

Пусть теперь $m_{0}>x_{1}^{d-1} x_{i_{s}} x_{n}^{r}$, тогда

$$
D^{R} M_{d+r}=\left\{m=x_{1}^{b_{1}+d-1} \cdots x_{n}^{b_{n}} \in A_{r+d}: b_{i_{1}}=b_{i_{2}}=\cdots=b_{i_{s}}=0, m \geqslant m_{0}\right\} .
$$

Представим $D^{A}\left(I_{d+r} \cup M_{d+r}\right)$ в виде $D^{A} I_{d+r} \cup B,|B|=\left|D^{R} M_{d+r}\right|$. Сопоставим каждому элементу из $D^{R} M_{d+r}$ элемент в $B$ по правилу:

$$
\begin{gathered}
x_{1}^{b_{1}+d-1} \cdots x_{n}^{b_{n}} \longmapsto x_{1}^{d-1} x_{s+1}^{c_{s+1}} \cdots x_{n}^{c_{n}}, \\
\left(c_{s+1}, \ldots, c_{n}\right)=\left(b_{1}, \ldots, \widehat{b}_{i_{1}}, \ldots, \widehat{b}_{i_{s}}, \ldots, b_{n}\right) \quad\left(b_{i_{j}} \text { пропушены }\right)
\end{gathered}
$$

в частности,

$$
m_{0}=x_{1}^{a_{1}} \cdots x_{n}^{a_{n}} \longmapsto m_{0}^{\prime}=x_{1}^{d-1} x_{s+1}^{a_{s+1}^{\prime}} \cdots x_{n}^{a_{n}^{\prime}} .
$$

Очевидно, что построенное отображение устанавливает взаимно однозначное сохраняюшее порядок соответствие между $D^{R} M_{d+r}$ и $B$, следовательно, $B=$ $x_{1}^{d-1} \cdot\left\{m=x_{s+1}^{b_{s+1}} \cdots x_{n}^{b_{n}} \in A_{r+1}: x_{1}^{d-1} m \geqslant m_{0}^{\prime}\right\}$.

Понятно, что $\Delta^{A}\left(D^{A}\left(I_{d+r}\right)\right)=x_{1}^{d-1} \cdot\left\{x_{1}^{r+2}, x_{1}^{r} x_{2}, x_{1}^{r} x_{3}, \ldots, x_{s} x_{n}^{r+1}\right\}$ состоит из всех мономов степени $d+r+1$, бо́льших $x_{1}^{d-1} x_{s+1}^{r+2}$, и $\Delta^{A}\left(I_{d+r}\right)=x_{1}^{d-1}$. $\left\{x_{i_{1}}, x_{i_{2}}, \ldots, x_{i_{s}}\right\} \cdot A_{r+1}$, следовательно, $\Delta^{A}\left(D^{A}\left(I_{d+r}\right)\right)=D^{A}\left(\Delta^{A}\left(I_{d+r}\right)\right)$. Кроме того,

$$
\begin{aligned}
& \Delta^{A}(B) \backslash \Delta^{A}\left(D^{A}\left(I_{d+r}\right)\right)=\left\{m=x_{1}^{d-1} x_{s+1}^{b_{s+1}} \cdots x_{n}^{b_{n}} \in A_{r+d+1}: m \geqslant m_{0}^{\prime} x_{n}\right\}, \\
& \Delta^{A}\left(D^{R} M_{d+r}\right) \backslash \Delta^{A}\left(I_{d+r}\right) \\
& \quad=\left\{m=x_{1}^{b_{1}+d-1} \cdots x_{n}^{b_{n}} \in A_{r+d+1}: b_{i_{1}}=b_{i_{2}}=\cdots=b_{i_{s}}=0, m \geqslant m_{0} x_{n}\right\} .
\end{aligned}
$$


Поэтому можно, как и выше, построить взаимно однозначное соответствие между множествами $\Delta^{A}\left(D^{R} M_{d+r}\right) \backslash \Delta^{A}\left(I_{d+r}\right)$ и $\Delta^{A}(B) \backslash \Delta^{A}\left(D^{A}\left(I_{d+r}\right)\right)$, следовательно, $\left|\Delta^{A}\left(D^{R} M_{d+r}\right) \backslash \Delta^{A}\left(I_{d+r}\right)\right|=\left|\Delta^{A}(B) \backslash \Delta^{A}\left(D^{A}\left(I_{d+r}\right)\right)\right|$, а так как $\left|\Delta^{A}\left(D^{A}\left(I_{d+r}\right)\right)\right|=\left|\Delta^{A}\left(I_{d+r}\right)\right|$, то

$$
\begin{aligned}
\left|\Delta^{A}\left(D^{R} M_{d+r} \cup I_{d+r}\right)\right| & =\left|\Delta^{A}\left(D^{R} M_{d+r}\right) \backslash \Delta^{A}\left(I_{d+r}\right)\right|+\left|\Delta^{A}\left(I_{d+r}\right)\right| \\
& =\left|\Delta^{A}(B) \backslash \Delta^{A}\left(D^{A}\left(I_{d+r}\right)\right)\right|+\left|\Delta^{A}\left(D^{A}\left(I_{d+r}\right)\right)\right| \\
& =\left|\Delta^{A}\left(D^{A}\left(M_{d+r} \cup I_{d+r}\right)\right)\right|,
\end{aligned}
$$

т.е. (6) доказано, а вместе с ним и (5) при $v \geqslant d$.

Если $v<d-1$, то (5) выполняется в силу теоремы Маколея для кольца $A$ $\left(I_{v}=\varnothing, v \leqslant d-1\right)$.

При $v=d-1$ имеем

$$
\left|\Delta^{R}\left(D^{R} M\right)\right|=\left|\Delta^{A}\left(D^{R} M\right)\right|-s \leqslant\left|\Delta^{A}(M)\right|-s \leqslant\left|\Delta^{R}(M)\right|
$$

(среднее неравенство - в силу теоремы Маколея в кольце $A$ ).

Теорема 4.11. Пусть $u, v \in A_{d}, u \geqslant v u I=L[u, v]$ - вполне лексический идеал в $A$. Теорема Маколея в $R=A / I$ выполняется тогда и только тогда, когда I удовлетворяет одному из следующих условий:

(a) $u=x_{1}^{d}$;

(b) $u=x_{1}^{d-1} x_{2}, v>x_{1}^{d-2} x_{2} x_{n}$.

ДокАЗАТЕльство. Если выполняется (а), то идеал $I$ является лекс-сегментньг, и по теореме 2.9 в $R$ вьполняется теорема Маколея.

Если выполняется (b), то в силу теоремы 4.1 ( $I$ - вполне лексический) имеем, что $v \leqslant x_{1}^{d-1} x_{n}$.

Пусть $M \subseteq R_{s}$. Докажем, что

$$
\left|\Delta^{R}\left(D^{R} M\right)\right| \leqslant\left|\Delta^{R}(M)\right| .
$$

Пусть $s<d-1$, тогда неравенство (7) выполняется в силу теоремы Маколея в кольце $A$.

Пусть $s=d-1$, тогда если $v=x_{1}^{d-1} x_{n}$, то $\left|\Delta^{R}\left(D^{R} M\right)\right|=\left|\Delta^{A}\left(D^{A} M\right)\right|-(n-1)$ и $\left|\Delta^{R}(M)\right| \geqslant\left|\Delta^{A}(M)\right|-(n-1)$, т.е. $\left|\Delta^{R}(M)\right| \geqslant\left|\Delta^{R}\left(D^{R} M\right)\right|$, а если $v=x_{1}^{d-2} x_{2} x_{k}$, $1<k<n$, то из $|M|=1$ следует, что $\left|\Delta^{R}\left(D^{R} M\right)\right|=1 \leqslant\left|\Delta^{R}(M)\right|$, а из $|M| \geqslant 2$ имеем $\left|\Delta^{R}\left(D^{R} M\right)\right|=\left|\Delta^{A}\left(D^{R} M\right)\right|-(n+k-2) \leqslant\left|\Delta^{R}(M)\right|$.

Пусть $s>d-1$. Обозначим $T=A /\left(I, x_{1}^{d}\right)$, тогда

$$
\left|\Delta^{R}\left(D^{R} M\right)\right|=\left|\Delta^{T}\left(D^{R} M \backslash x_{1}^{s}\right)\right|+1 \leqslant\left|\Delta^{R}(M)\right| .
$$

Пусть теперь в $R$ выполняется теорема Маколея, докажем выполнение условий (a) и (b). В силу теоремы 2.10 имеем, что $u \geqslant x_{1}^{d-1} x_{n}$.

Если $u=x_{1}^{d-1} x_{k}, k \geqslant 3$, то $\left|\Delta^{R}\left(x_{1}^{d}\right)\right| \geqslant k-1 \geqslant 2$, и в силу того, что $I$ - вполне лексический, $\left|\Delta^{R}\left(x_{1}^{d-1} x_{2}\right)\right|=1$, т.е. $\left|\Delta^{R}\left(x_{1}^{d}\right)\right|>\left|\Delta^{R}\left(x_{1}^{d-1} x_{2}\right)\right|-$ противоречие, значит, $u \geqslant x_{1}^{d-1} x_{2}$. Осталось доказать, что если $u=x_{1}^{d-1} x_{2}$, то $v>x_{1}^{d-2} x_{2} x_{n}$.

Пусть $v \leqslant x_{1}^{d-2} x_{2} x_{n}$, тогда $\left|\Delta^{R}\left(x_{1}^{d-2} x_{2}\right)\right|=0,\left|\Delta^{R}\left(x_{1}^{d-1}\right)\right|=1$-противоречие, значит, $v>x_{1}^{d-2} x_{2} x_{n}$. 
Теорема 4.12. Пусть $u, v \in A_{d}, u \geqslant v u I=L[u, v]-$ лексический идеал в А. Теорема Маколея в $R=A / I$ виполняется тогда и только тогда, когда I удовлетворяет одному из следующих условий:

(a) $u=x_{1}^{d}$;

(b) $u=x_{1}^{d-1} x_{2}, v>x_{1}^{d-2} x_{2} x_{n}$;

(c) $u=x_{1}^{d-1} x_{k}, k>2, v>x_{1}^{d-2} x_{2}^{2}$.

ДокАЗАТЕЛЬство. Если выполняется (а), то идеал $I$ является лекс-сегментньм, и по теореме 2.9 в $R$ вьполняется теорема Маколея.

Пусть выполняется (b). Если $v \leqslant x_{1}^{d-1} x_{n}$, то в силу теоремы $4.1 I$ является вполне лексическим, следовательно, по теореме 4.11 в $R$ выполняется теорема Маколея. А если $v>x_{1}^{d-1} x_{n}$, то по теореме 4.10 в $R$ выполняется теорема Маколея.

Если выполняется (с), то теорема Маколея в $R$ вьполняется в силу теоремы 4.10 .

Пусть теперь в $R$ выполняется теорема Маколея. По теореме $2.10 u \geqslant x_{1}^{d-1} x_{n}$. Если $u=x_{1}^{d-1} x_{2}$, но $v \leqslant x_{1}^{d-2} x_{2} x_{n}$, то $\left|\Delta^{R}\left(x_{1}^{d-1}\right)\right|=1>\left|\Delta^{R}\left(x_{1}^{d-2} x_{2}\right)\right|=0-$ противоречие.

Если, наконец, $u=x_{1}^{d-1} x_{k}, k>2$, но $v \leqslant x_{1}^{d-2} x_{2}^{2}$, то $\left|\Delta^{R}\left(x_{1}^{d}\right)\right|=k-1>$ $\left|\Delta^{R}\left(x_{1}^{d-1} x_{2}\right)\right|=k-2$ - противоречие.

\section{Список литературы}

1. Macaulay F. S. Some properties of enumeration in the theory of modular systems // Proc. London Math. Soc. 1927. V. 26. P. 531-555.

2. Clements G.F., Lindström B. A generalization of a combinatorial theorem of Macaulay // J. Combin. Theory. 1969. V. 7. P. 230-238.

3. De Negri E., Herzog J. Completely lexsegment ideals // Proc. Amer. Math. Soc. 1998. V. 126. P. $3467-3473$.

4. Eliahou S., Kervaire M. Minimal resolutions of some monomial ideals // J. Algebra. 1990. V. 129. P. $1-25$.

Московский государственньй

Поступила в редакцию университет им. М.В. Ломоносова 